\title{
An Extended Lagrangian Method
}

\author{
Meng-Sing LIOU \\ Internal Fluid Mechanics Division, NASA Lewis Research Center, Cleveland, Ohio 44135
}

Received June 1, 1993; revised July 21, 1994

\begin{abstract}
A unique formulation of describing fluid motion is presented The method, referred to as "extended Lagrangian method," is interesting from both theoretical and numerical points of view. formulation offers accuracy in numerical solution by avoiding numerical diffusion resulting from mixing of fluxes in the Eulerian description. The present method and the Arbitrary LagrangianEulerian (ALE) method have a similarity in spirit-eliminating the cross-streamline numerical diffusion. For this purpose, we suggest a simple grid constraint condition and utilize an accurate discretization procedure. This grid constraint is only applied to the transverse cell face parallel to the local stream velocity, and hence our method for the steady state problems naturally reduces to the streamlinecurvature method, without explicitly solving the steady streamlinecoordinate equations formulated a priori. Unlike the Lagrangian method proposed by Loh and Hui which is valid only for steady supersonic flows, the present method is general and capable of treating subsonic flows and supersonic flows as well as unsteady flows, simply by invoking in the same code an appropriate grid constraint suggested in this paper. The approach is found to be robust and stable. It automatically adapts to flow features without resorting to clustering, thereby maintaining rather uniform grid spacing throughout and large time step. Moreover, the method is shown to resolve multi-dimensional discontinuities with a high level of accuracy, similar to that found in one-dimensional problems. 1995 Academic Press, Inc.
\end{abstract}

\section{INTRODUCTION}

It is well known that fluid motion can be specified by either the Eulerian or Lagrangian description. Most CFD developments over the last three decades have been based on the Eulerian description and considerable progress has been made. In particular, the upwind methods, inspired and guided by the work of Gudonov [1], have met with a great deal of success in solving fluid flows, especially where discontinuities exist. However, this shock capturing property has proven to be accurate only when the discontinuity is aligned with one of the grid lines since most upwind methods are strictly formulated in a one-dimensional framework and only formally extended to multi-dimensions. Consequently, the attractive property of crisp resolution of these discontinuities is lost. Even though research on genuine multi-dimensional approaches has recently been undertaken by several leading researchers, they are nevertheless still based on the Eulerian description.
Recently, Loh and Hui [2] have convincingly demonstrated that a Lagrangian formulation can capture a contact discontinuity crisply, which is difficult to achieve by Eulerian formulation without resorting to some special treatment such as subcell resolution. Further developments have been carried out by Loh and Liou to solve real gas problems [3] and three-dimensional supersonic problems [4]. The 3D extension is not so trivial, and in fact it involves somewhat tricky definition of cell movement and approximate solution of the multi-dimensional Riemann problem. Several interesting 3D problems that have not been attempted previously were calculated and again as in the 2D case, high accuracy was achieved for resolving very complex shock-shock interactions. This method employs the point of view that it strictly follows the fluid particles released at some initial time line. The streamlines become a "time-like", coordinate and are used also for identifying particles. Therefore the method is naturally suitable for supersonic steady flow. No grid generation is needed a priori since the grid is part of the solution, viz., new grid lines are obtained as the solution marches in the "time-like" direction. Unfortunately, restriction to supersonic flows only limits the use of the method. To include the subsonic regime requires substantial conceptual changes.

Numerically solving subsonic flows using the strict Lagrangian concept becomes an excessive obstacle. Numerous researchers at Los Alamos, making substantial contributions to this subject, have proposed the so-called Arbitrary LagrangianEulerian (ALE) method, for example $[5,6,7]$ and others. Their method basically consists of two phases, namely Lagrangian and rezone phases. $A$ third and implicit phase, similar to the Implicit Continuous-Fluid Eulerian (ICE) method [8] is inserted in the Lagrangian phase to allow for an efficient solution of flows at all speeds [5]. The method permits optimal use of grid, which may move with the fluid (Lagrangian mode), or remain fixed with fluid moving through the cells (Eulerian mode), or move in any other prescribed manner (Arbitrary LagrangianEulerian mode). This flexibility allows for the calculation of flows involving curved or moving boundaries and makes possible calculations with minimum computational diffusion without excessive grid distortions.

Although the concept for the Lagrangian approach and the equations to be solved are naturally very appealing, the procedure involving geometrical and variable constructs for the dis- 

crete system becomes rather complicated, see [5-7]. Tracking the Lagrangian cells requires movement of cell vertices which must be done by interpolating variables and coordinates. It is well known that most multidimensional Lagrangian calculations can only be continued for a finite time before the mesh is destroyed by "tangling," or crossing of mesh lines [7]. The rezone phase is then added to prevent grid lines from crossing each other or grid tangling. Loss of accuracy, because of accounting for exchange of materials among the surrounding cells, can be inflicted through the continuous geometry and flow interpolations in response to the fluid particle motion [7]. In this regard, the paper can be thought of contributing a concept of moving the grid for this rezone phase in a specific way so that numerical diffusion at the cross stream faces remains vanished, as in the case of the strict Lagrangian approach. A more detailed comparison of the present approach and the ALE method is deferred to Section 3.

Physically fluid particles at subsonic speed seem to adjust to motion and to surrounding (geometric or physical) constraints quickly and graciously, in particular sensing the upstream-propagating influences. Thus, a key to the success of a numerical Lagrangian procedure lies in how to properly and instantaneously feed these upstream-propagating waves to the particles, while tracking them. It is indeed a very challenging research topic that motivates us to begin this exploratory investigation. This paper presents the salient features of the method, referred to as "extended Lagrangian method." For flows at all speed regimes including purely subsonic and mixed flows, we demonstrate the advantages of the method over the Eulerian description, with focuses on important features commonly seen in compressible flows, such as shocks, expansion waves, slip surfaces, and interactions among them. We summarize in the following some interesting features of the present extended Lagrangian method. Specific advantages of the Lagrangian approach over the Eulerian one are given in Section 2 and additional advantages of the present extended Lagrangian approach in Section 4.

1. The solution adapts to the flow variations (smooth or sudden), notably shocks and contacts, and as such it can be regarded as an automatic procedure for solution adaptation.

2. Unlike the conventional adaptation techniques, there is no need for clustering grid points near the discontinuities. Very uniform grid can be maintained and in fact can also achieve orthogonality easily by construction. Thus discretization accuracy does not deteriorate. Since streamlines do not cross, grid singularity or negative volume certainly will not occur.

3. As will be seen later, the shock capturing quality in $2 \mathrm{D}$ is comparable to that found in the 1D problem. This suggests that the present approach can be viewed as an alternative to the current genuine multi-dimensional approach.

4. The contact discontinuity can be resolved crisply, since it is a streamline and as such no numerical diffusion is introduced due to fluid crossing the cell face.
The rest of the paper is organized as follows. In Section 2 we compare differences of Eulerian and Lagrangian descriptions of fluid motion, with an emphasis on the numerical aspects. Some current methods relevant to the scope of the present paper concerning the Lagrangian and streamline-coordinates approaches are commented on in Section 3. Section 4 outlines the key elements in formulating the present extended Lagrangian method for solving subsonic flows by retaining the advantageous features of the Lagrangian approach. A detailed formulation is then given in Section 5. Section 6 describes the grid motion of the present "extended Lagrangian" method. In Section 7 we briefly include the discretization method to complete the numerical procedure. Finally, in Section 8 we demonstrate the advantages of the proposed method by displaying solutions of flows at all speed regimes, containing various interesting features.

It is noted that for the following sections, especially Sections 2 and 5 , while some of the statements may be well known, thus repetitive, to some readers, they are presented primarily with different concerns and motivations in mind, namely numerical viewpoint. In this way, a systematic and self-contained description can be made clear and we opt for elaboration rather than conciseness.

\section{EULERIAN VS LAGRANGIAN DESCRIPTION}

By definition, the Eulerian description oberves at fixed locations the flow properties as fluid particles pass by. This has a close relation to the conventional computation approach in that each fixed grid point can be thought of as an observing stationcorresponding to probes in measurement. With this approach, the meshes are generated mainly based on the geometry constraint, with little regard given to the motion of fluid. Naturally, the grid lines will seldom coincide with fluid path lines. Even when grid lines are clustered near high-gradient regions using conventional adaptation techniques, they are not aligned with the particle path. A good example is the shock wave along which grid lines are densely distributed, and with which streamlines make a nonzero angle, since the fluid will always pass through, not along, the shock wave. The angle is usually oblique in multidimensional flows. Consequently, the Eulerian approach has several severe numerical effects on the solution accuracy:

1. Fluid particles are free to cross the grid line, thereby bringing (convecting) with them numerical mixing and diffusion across the cell interface.

2. This numerical diffusion is only associated with the error resulting from approximating the convective terms.

3. A contact/slip or shear layer is smeared ever increasingly with time and distance, unless some detection and special treatment techniques are employed. See [9] for example.

Items 1 and 2 may appear to be two sides of a coin, indeed they are closely related on the discrete level. Item 1 can be a purely physical process if one were able to represent the cell- 
face fluxes precisely as if in the continuous (differential) level. Thus, item 3 is the consequence of 1 , in which the convection is represented approximately by a numerical procedure. It then becomes clear that the numerical procedure with minimum diffusion can be designed so that either the free crossing (exchange) of mass is entirely restricted or the convective flux of the continuous system is precisely duplicated at the cell face. The latter proposition would seem impossible to do since the idea of discretization is to assume a function by an approximate valid in a finite domain, in which the approximate would tend to the function as the domain vanishes. Clearly the choice is the former.

In spite of numerical diffusion resulting from approximation of convective terms, the Eulerian description does offer convenience and simplicity both conceptually and geometrically. The grid can be constructed regularly, simply based on geometry constraints and with little attention paid to the flow features. To enhance accuracy, grid adaptation is often applied to regions of high gradients. However, the concept of adapting to high gradients inevitably results in a skewed and distorted grid. This feature in turn will become more troublesome as two or more high-gradient areas intersect.'

It is quite safe to say that during the last three decades CFD algorithm researchers have primarily concentrated on developing better (more accurate/robust/efficient) ways to deal with the convective terms, which exist only in the Eulerian formulation. Consequently, much success has been achieved, perhaps to the point of near perfection and little return could be gained. Unfortunately, inaccuracy due to numerical diffusion (mixing) in forming the interface numerical fluxes still exists and becomes more exaggerated in multi-dimensional problems. On the other hand, since the convective terms do not explicitly appear in the Lagrangian formulation, the numerical mixing automatically disappears in the flux evaluation, rendering the Lagrangian approach attractive with respect to this viewpoint. However, other technical barriers have surfaced and discouraged researchers from further pursuing development of methods for this approach. In what follows, we shall detail the concept of the Lagrangian approach from the viewpoint of numerical solution. The differences between the two descriptions will then become obvious.

The Lagrangian description, by definition, states the motion and properties of given fluid particles as they travel to different locations. In particular, since the particle path in steady flow coincides with the streamline, no fluid particles will cross the streamline. In other words, while staying in contact, neigh-

\footnotetext{
The unstructured grid approach will also face the same inaccuracy issue in approximating the convective terms, so long as the Eulerian description is adopted. To reduce the numerical diffusion error, adaptive remeshing techniques can be employed in both structured and unstructured grids by adding more and more points, i.e., finer and finer meshes, near the high-gradient regions. This viewpoint in fact deals with item 2 in the above comment-reduction of the the cross-streamline diffusion by shrinking the grid size. However, the remeshing comes at the expenses of computation and data structure.
}

boring streams will not mix via convection, except in the molecular level where the physical molecular diffusion takes place. Therefore, the following numerical consequences can be realized:

1. No numerical diffusion is introduced across the cell interface since the computational cells follow the streamline, leading to a crisp resolution of contact/slip surfaces

2. Fluid particles change motion (direction/speed) only as warranted, e.g.. as shock or expansion waves are encountered. In other words, streamlines will bend, converge, or diverge only as situations demand. As a result, the shock capturing quality in 2D is comparable to that found in the ID problem, suggesting the present approach as an alternative to the genuine multi-dimensional approach.

3. This description gives a realistic depiction of flow behavior; cells of same $j$-index form a streamline that is identifiable with flow visualization.

The notion of using a Lagrangian approach to describe flow is not new. In fact, the very essence of following fixed particles also presents mathematical complexity to the approach, thereby limiting its scope of success. With the help of the new Lagrangian formulations, numerical solution can be as easily obtained as for the Eulerian approach, only with the additional distinct advantages as stated above. In the following, we shall first review some current numerical procedures based on the Lagrangian approach, commenting about their strengths and weaknesses. Then we will focus on the applicability to the more difficult problem, namely the subsonic regime.

\section{REVIEW OF RELEVANT APPROACHES}

The present method, as will become clear in what follow, share several common concepts with the ALE method and the streamline-coordinate approaches. Yet, it also differs in either concept or procedure from the above two. Thus it is useful to give a brief discussion on these methods.

\subsection{Lagrangian/ALE Methods}

The Arbitrary Lagrangian-Eulerian Technique (ALE) perhaps is the most well-known Lagrangian formulation in use at the present time. The technique, initially conceived and developed at Los Alamos during the 1970s, was implemented in several production codes such as CAVEAT and KIVA $[10,11]$, and the others. For the purpose of discussion, we will briefly summarize some of the key features in this technique; for a complete description of the procedure, the reader is referred to Refs. $[5$, $6,10,111$. The ALE method, while evolving throughout the years, maintains some basic constructs. It uses the staggered grid on which velocity components are stored at the cell vertices, thus directly giving displacements of vertices, and other variables at the cell center. The method basically consists of two phases of numerical procedures, namely Lagrangian and 
rezone phases, using the time splitting concept. A third and implicit phase, similar to the Implicit Continuous-Fluid Eulerian (ICE) method [8] is added in the Lagrangian phase to allow for efficient solution of flows at all speeds [5]. The method permits optimal use of grid, which may move with the fluid (Lagrangian mode), or remain fixed with fluid moving through the cells (Eulerian mode), or move in any other prescribed manner (Arbitrary Lagrangian-Eulerian, ALE, mode). This flexibility allows for the calculation of flows involving curved or moving boundaries and makes possible calculations with minimum computational diffusion without excessive grid distortions.

The Lagrangian approach and the equations to be solved are indeed very appealing conceptually. Tracking the Lagrangian cells requires movement of cell vertices which in turn involves interpolation of geometrical and variables data. Premature tangling failure can result from numerical errors such as discretization errors and nonuniform mesh, the latter being the subject of investigation in [7]. To prevent this grid difficulty, the rezone phase is added to regularize the cell distortion in a prescribed manner-optimal choice for controlling the grid distortion would be problem dependent. Loss of accuracy, because of accounting for exchange of materials among the surrounding cells in the ALE mode, can be inflicted through the continuous geometry and flow interpolations in response to the fluid particle motion. As a result, spurious error produced by this procedure can feed to the cycle of grid irregularity and tangling, see [7].

Recently a new Lagrangian Formulation was proposed by Hui and Van Roessel [12]. The inviscid conservation laws are transformed by using stream functions and Lagrangian time as independent variables. The stream functions serve to identify particles, while Lagrangian time represents time-like coordinate. Under this formulation, geometry conservation is enforced and each cell is literally a fluid particle. The grid movement, although often distorted, is obtained as part of the solution in the time-like (equivalently space) marching process from the derived geometrical conservation laws. The method has been shown to allow for extremely sharp resolution of contact discontinuities by Loh and Hui [2] in 2D and Loh and Liou [4] in 3D problems. Multi-dimensional discontinuities are resolved with the same level of accuracy as their one-dimensional counterparts, indicating that the Lagrangian formulation inherently includes multi-dimensional flow characteristics. However, a severe limitation restricts the validity of the formulation [2-4] to only supersonic flows because the formulation is based on the use of the time-like coordinate. Thus, extention to subsonic flows based on the same framework appears impossible.

Also, when a contact/slip surface exists initially, the fluid particles on either side of it may start separating from each other and eventually lose contact, disallowing interactions of fluxes (pressure only) from these fluid cells. Loh and Hui [2] applied a quick fix by subdividing the Lagrangian cells in a manner that the "time" increment (i.e. the sub-cell size) is adjusted to permit the cells remain in contact. Now, the strict "Lagrangian" concept begins to be relaxed since the sub-cells are only part of the initial cells, albeit belonging to the same identity. A formal transformation from the "Lagrangian time" ( $\tau$-form) to the "Lagrangian" distance ( $\lambda$-form) was suggested by Hui and Zhao [13]. Conceptually, this draws close similarity to the steady streamline-coordinate method [14-18] in which one of the coordinates is a streamline. In fact, the discrete equations written in finite volume form from both formulations are identical for steady supersonic flows. Naturally, differences among these methods eventually appear as choices for representing fluxes are made. Hence, it is relevant to discuss some aspects of the so-called streamline-coordinate method.

\subsection{Streamline-Coordinate Method ${ }^{2}$}

Both the entropy and total enthalpy are conserved along streamlines if the flow is steady, inviscid, and adiabatic. Thus, there are advantages in exploiting these exact properties in the analysis by choosing streamlines as one of the coordinates. In the classical analysis, the other coordinate are defined to be the normals to the streamlines, this system is commonly known as Streamline Curvature [19], or Intrinsic, or Natural Coordinates [20]. Thus, in a two-dimensional irrotational steady flow, this coordinate system comprises of coodinates coinciding with the streamlines and equipotential lines. Being as natural as the intrinsic coordinate system may be, reports about solutions based on these coordinates, however, have been scarce in the literature. The streamline curvature method was popular in engine industry for calculating subsonic flows in turbomachinery. Nonconservative steady differential equations valid along and normal to the streamline are used and solved numerically by iterative relaxation procedures [14-16]. Making use of the advances in the Eulerian approaches, Giles and Drela [17, 18] solved the discrete approximate equations of the steady streamline-coordinate equations in conservation form. Each 2D quadrilateral cell is defined such that there is no mass across two of the four sides. Hence the mass and energy fluxes in each cell along the streamline coordinate are particularly simple. The only contribution to the steady-state equations from the streamline faces comes from the pressure contribution to the momentum equations. An auxiliary pressure relation must be assumed in order to close the resulting system. The grid positions are not known a priori, and must be solved as part of the solution. Supersonic and transonic flows with shocks were solved using the artificial compressibility for maintaining stability [18]. A common feature in all these developments is the use of steady-state equations which are explicitly written on the basis of the streamline coordinates. This does not necessarily offer the best choice for resolving practical flows from the numerical point of view. The streamlines may be tightly bundled with large curvature, as in the vortical flow, or may run into singularities, such as separation and reattachment points.

\footnotetext{
2I thank one of the referees who kindly brought my attention to the literature on the streamline curvature method. This section is the result of surveying some past works on the subject.
} 
In these situations, we speculate that the grid (geometrical) and flow variables, as closely coupled, could result in numerical difficulty, eventually terminating solution.

As stated in the last section, the so-called $\lambda$-form of Hui and Zhao [13] for the steady supersonic flow indeed falls in the category of the streamline-coordinate method. While a rigorous algebraic manipulations has been carried out in the differential equation level, the discrete equations could be derived much directly from the integral consideration, as done in $[17,18]$ and later in Section 5. In their formulation, the $\lambda$-coordinate coincides with the streamline and thus only pressure acts on the faces along $\lambda$. The grid is obtained like the other variables in a space-marching fashion. From the forgoing discussion, it is clear that the methods classified in Sections (3.1) and (3.2) are identical in the case of steady supersonic flows and share the same objective for eliminating the numerical diffusion associated with fluid crossing cell boundaries. Having said this, we note that significant differences between approaches in Sections (3.1) and (3.2) exist for arriving at this objective, these differences mainly born out of numerical consideration. In this paper, we present an approach that can be seen to combine advantages of the above two: numerical flexibility of (3.1) for moving the grid and vanishing numerical diffusion (convective error) across the streams. Other distinct features will also be brought out in Section (5.3).

In what follows we will first give the basic ideas for extension for subsonic flows in the next section and then describe detailed steps in Section 5.

\section{EXTENSION TO SUBSONIC FLOWS}

A key element in the subsonic flow is the existence of the upstream-propagating wave. Thus, the existence of a body located downstream is transmitted to the oncoming fluid particles via this wave so that the particles can change motion accordingly. This immediately implies that we must abandon the timelike formulation cited above since it is only suited for pure initial value problems, such as supersonic flow where no influence comes from downstream. Next, we must also abandon the idea of following a fixed particle, at least for the steady flows. Alternatively, we consider the steady streamlines as a set of lines that are occupied by particles released at the same location, different times and yet treated indistinguishably. The upstreampropagating influence is felt through the downstream particles on the same streamline in order to satisfy the governing conservation equations and boundary conditions in question. By describing fluid motion along streamlines, we allow fluids to maintain their identity without tracking each specified particle. This definition is of course broader than and is a sufficient condition to the Lagrangian description, which follows motion of fluids of specific identity. Consequently, the present method is termed extended Lagrangian method. In other words, our approach (which boils down to the constraint given later in Eq. (10)) includes the strict Lagrangian description as a special case. The net result is that we retain the essential beauty of the Lagrangian description that introduces no or minimal numerical diffusion across streamlines, but also at the same time we avoid its difficulty in grid tangling. On the other hand, we extend the streamline-coordinate method by not solving equations written in streamline coordinates, which are not known a priori, and by not using the auxiliary pressure relation $[17,18]$.

\section{FORMULATION}

To facilitate the description, let us first define the notation for the relevant variables in the 3D Euler equations. The physical variables in a phase space of dimension 5 are denoted by a boldface uppercase letter or column vector whose elements are denoted by lowercase letters.

$$
\mathbf{U}=\left(\begin{array}{c}
\rho \\
\rho u \\
\rho v \\
\rho w \\
\rho e_{1}
\end{array}\right), \quad \mathbf{U}_{c}=\left(\begin{array}{c}
\rho \\
\rho u \\
\rho v \\
\rho w \\
\rho h_{1}
\end{array}\right) \text {, }
$$

where $e_{t}=e+0.5\left(u^{2}+v^{2}+w^{2}\right)$ and $h_{t}=e_{t}+p / \rho$. The geometrical vectors in physical (Cartesian) space of dimension 3 are denoted by an overhead arrow " $\rightarrow$ ". The fluid velocity is

$$
\vec{V}=u \vec{i}+v \vec{j}+w \vec{k},
$$

and the normal vector of the boundary surface of a control volume

$$
\vec{S}=s_{x} \vec{i}+s_{y} \vec{j}+s_{z} \vec{k} .
$$

The inviscid fluxes in 3D physical space are compactly written as

$$
\begin{array}{r}
\overrightarrow{\mathbf{F}}=\left(\begin{array}{c}
\rho \\
\rho u \\
\rho v \\
\rho w \\
\rho h_{i}
\end{array}\right) \vec{V}+\left(\begin{array}{c}
0 \\
p \vec{i} \\
p \vec{j} \\
p \vec{k} \\
0
\end{array}\right)=\mathbf{U}_{c} \vec{V}+\overrightarrow{\mathbf{P}}, \\
\text { where } \overrightarrow{\mathbf{P}}=\left(\begin{array}{c}
0 \\
p \vec{i} \\
p \vec{j} \\
p \vec{k} \\
0
\end{array}\right) .
\end{array}
$$

The first term in $\overrightarrow{\mathbf{F}}$ is the flux of $\mathbf{U}_{c}$ convected by the fluid velocity $\vec{V}$ and the second term simply the pressure flux 

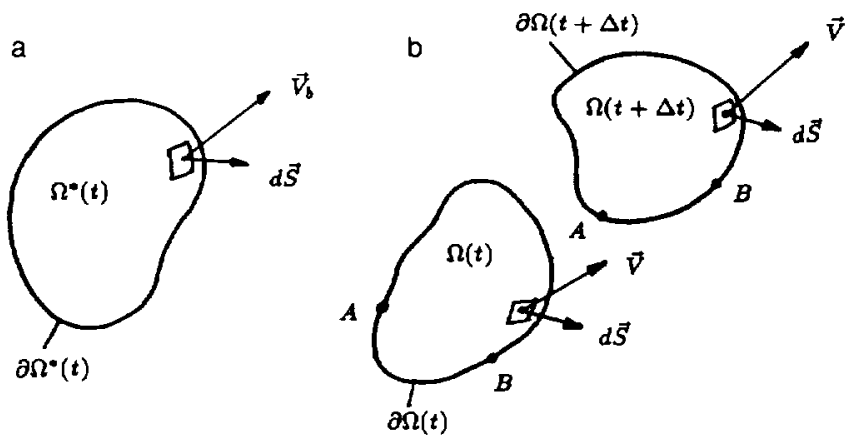

FIG. 1. Definition of a control volume and material volume. (a) Control volume. denoted by "**", may be nonstationary, e.g., on rotating frame. (b) Material volume, $\dot{V}_{b}=\dot{V}$

For the following discussion, it is instructive to review some basic concepts used to describe fluids. Even though some statements may seem redundant to some readers, we attempt to arrive at essential conclusions primarily from the numerical point of view and for the sake of self-containedness. The derivation given below is systematic and, to my knowledge, is not found elsewhere.

To make the presentation self contained, a slight preliminary is useful. It is understood that the fluid has been considered to be a continuum. A convenient concept within continuum mechanics for describing a fluid motion is that of control volume. In Fig. 1, let $\Omega^{*}(t)$ be a moving volume with bounding surface $\partial \Omega^{*}(t)$; the local boundary velocity is $\vec{V}_{b}$. The volume is arbitrary and in general need not be identified with either physical boundary or specific motion of the fluid in $\Omega^{*}$. Such a volume is called control volume. A special type of control volume is called material volume, denoted by $\Omega(t)$, consisting of a collection of matter of fixed identity enclosed by a material surface, denoted by $\partial \Omega(t)$, of which every point moves with the local fluid velocity $\vec{V}$. (See also Fig. 1.) If the volume $\Omega(t)$ is shrunk to a point, the resulting material volume is called a fuid particle. Consequently, the fluid properties of the fluid

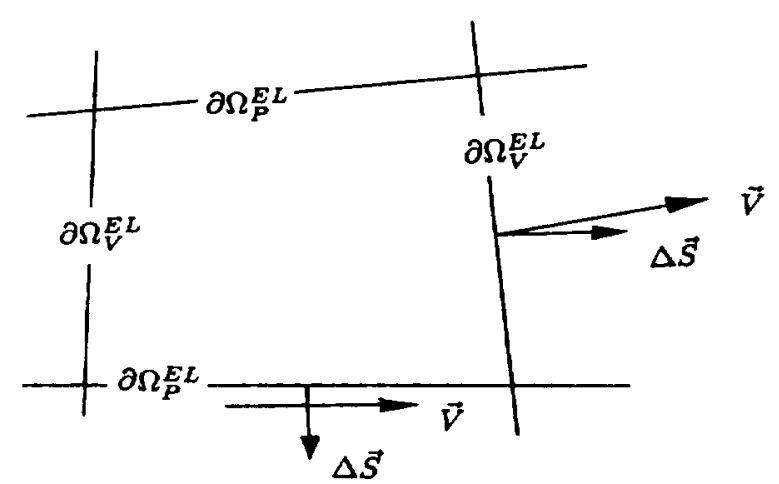

FIG. 2. Definition of an Extended Lagrangian volume: the cell boundary $\partial \Omega \Omega_{p}^{\mathrm{EL}}$ is parallel to the fluid velocity $\vec{v}$ particle can be described mathematically in terms of space and time.

Under the assumption of the continuum mechanics, let $\chi$ be any continuous function, such as the density. The Reynolds transport theorem [21] gives the time rate of change of the "content" $\chi$ of $\Omega^{*}$ :

$$
\frac{d}{d t} \int_{\left[\Omega^{*}(t)\right.} \chi d v=\int_{\left(2^{*}(I)\right)} \frac{\partial \chi}{\partial t} d v+\int_{i \Omega^{2}(t)} \chi \vec{V}_{b} \cdot d \vec{S}
$$

where the element surface $d \vec{S}$ of $\Omega *$ is moving with the velocity $\vec{V}_{h}$. Note that $\vec{V}_{b}$ may vary over the surface $\dot{d} \Omega^{*}$. Again, a special case is when the theorem is applied to the material volume $\Omega(t)$ with $\vec{V}_{h}=\vec{V}$.

The conservation laws (neglecting viscous fluxes for simplicity, without loss of generality in describing the approach) can be conveniently expressed over an arbitrary control volume $\Omega^{*}(t)$ in integral form:

$$
\begin{array}{r}
\frac{d}{d t} \int_{\mathbb{N}^{2}(t)} \mathbf{U} d v+\int_{\dot{d} \mathbf{R}^{*}(t)}\left[\mathbf{U}_{(}\left(\vec{V}-\vec{V}_{h}\right)+\overrightarrow{\mathbf{P}}_{h}\right] \cdot d \vec{S}=0 \\
\text { with } \overrightarrow{\mathbf{P}}_{b}=\overrightarrow{\mathbf{P}}+\left(\begin{array}{c}
0 \\
0 \\
0 \\
0 \\
p \vec{V}_{b}
\end{array}\right) .
\end{array}
$$

From the above equations, three fundamentally different approaches can result.

\subsection{Eulerian Description}

The Eulerian description assumes that the observer stays stationary with respect to the chosen frame of reference (e.g., inertial system). This requires:

$$
\vec{V}_{b}=0 \quad \text { and } \quad \Omega * \neq \Omega *(t) .
$$

That is, the control volume is fixed in time.

With the application of the Reynolds transport theorem Eq. (4), Eq. (5) is reverted to the familiar integral form:

$$
\int_{\Omega^{k}} \frac{\partial \mathbf{U}}{\partial t} d v+\int_{i t^{k}}\left[\mathbf{U}_{c} \vec{V}+\overrightarrow{\mathbf{P}}\right] \cdot d \vec{S}=0,
$$

where the superscript " $E$ " is used to denote Eulerian frame of reference. In the discrete version of the above equation, each 


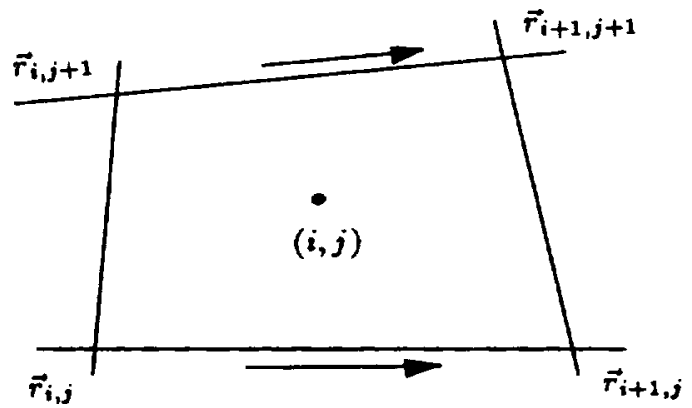

FIG. 3. Generating an Extended Lagrangian computational cell so that the cell boundary $\left(\vec{r}_{i+1, j+1}-\vec{r}_{i, j+1}\right)$ is parallel to $\vec{V}$

cell represents a control volume and is not moving in time, even though the flow may be unsteady. Note that the case in which an observer is fixed to a non-inertial frame, e.g., on rotating machines, also belongs to the Eulerian description.

\subsection{Lagrangian Description}

According to the strict definition, the Lagrangian description requires that the volume $\Omega^{*}(t)$ move with the instantaneous
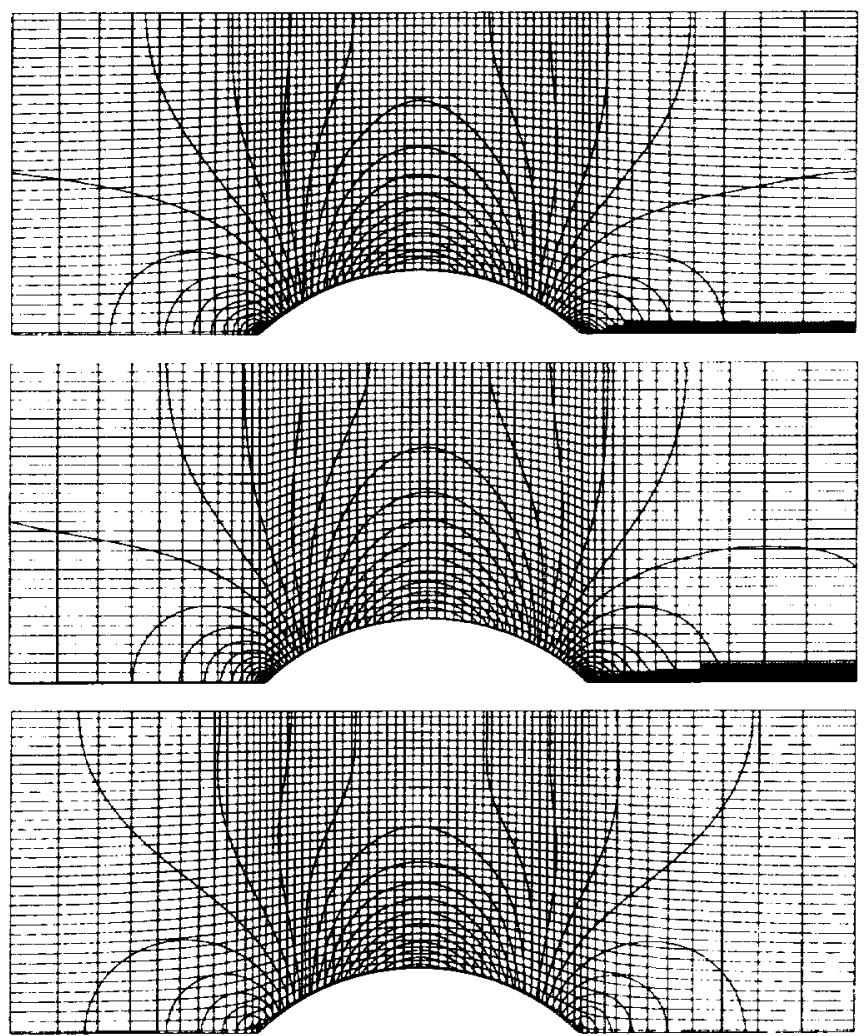

FIG. 4. Mach contours for $M_{x}=0.4,20 \%$ bump flow; (a) Lagrangian solution $(134 \times 70$ grid $)$, shaded area indicates numerical wake, (b) Eulerian solution ( $266 \times 138$ grid), and (c) pressure contours. noting normal behavior in the wake region. (Contour levels: $M_{\mathrm{m} \mid \mathrm{n}}=0.0, M_{\mathrm{max}}=0.8, \Delta M=0.02$.) fluid velocity and be identified as the material volume $\Omega(t)$ (see also Fig. 1). That is,

$$
\vec{V}_{h}=\vec{V} \text { for all } \forall t>0 \quad \text { and } \quad \Omega *(t)=\Omega(t) .
$$

And the conservation laws are simplified to

$$
\frac{d}{d t} \int_{S(t) t} \mathbf{U} d v+\int_{H(S(t)} \overrightarrow{\mathbf{P}}_{b} \cdot d \vec{S}=0 .
$$

Clearly, the pressure remains as the only contribution to the flux on the surface. This renders extremely simple calculation of the time-rate of change of $\mathbf{U} d v$-involving only the pressure acting on the bounding surfaces, only if $\Omega(t)$ is known precisely. However, the trajectory of the vertices is the part that often causes difficulties, resulting in large deformation or irregularity. (See [5-7].) Nevertheless, this is a very intriguing idea that avoids the nonlinearity in the equation of motion, thus reducing many difficulties associated with the convective terms that exist only in the Eulerian viewpoint. Such nice properties unfortunately have not been able to outweigh the drawbacks (see Introduction) and gain favorable reception over the Eulerian approach.

In the following, we propose a new approach that retains essential advantages of the above two approaches.

\subsection{Extended Lagrangian Description}

Close investigation of the surface integral reveals that the convective term can be eliminated also by requiring the constraint:

$$
\left(\vec{V}-\vec{V}_{b}\right) \cdot \vec{S}=0 .
$$

This is the most general condition to vanish numerical diffusion resulting from convection. It amounts to either of the statements: (a) alignment of cell surfaces with the fluid relative velocity $\left(\vec{V}-\vec{V}_{b}\right)$-reducing to streamline coordinates for steady-state flow where $\vec{V}_{b}=0$, or (b) $\vec{V}=\vec{V}_{b}$, which is the strict Lagrangian description. Since (b) is included in Eq. (10) as a special case, our method is thus termed the "Extended Lagrangian Method", and Eq. (10) is termed constraint "EL".

It should be kept in mind that the condition, Eq. (10), is also valid at any instant, thus applicable to unsteady flow problems. Equation (5) becomes, as the constraint EL is imposed,

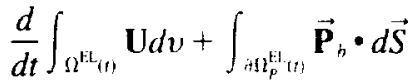

$$
\begin{aligned}
& +\int_{\partial \Omega_{v}^{\mathrm{E}}(t)}\left[\left(\vec{V}-\vec{V}_{b}\right) \mathbf{U}_{c}+\overrightarrow{\mathbf{P}}_{b}\right] \cdot d \vec{S}=0 .
\end{aligned}
$$

The control volume now is denoted by superscript "EL" to indicate the present description. The surface $\partial \Omega^{\mathrm{EL}}$ is comprised of two types, $\partial \Omega^{\mathrm{EL}}=\partial \Omega_{P}^{\mathrm{EL}} \cup \partial \Omega_{\psi}^{\mathrm{EL}}$, where $\partial \Omega_{P}^{\mathrm{EL}}$ coincides 


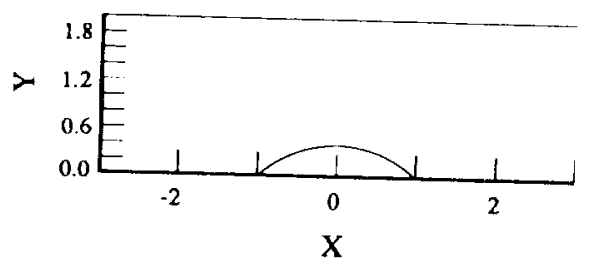

20\% BUMP ON A CHANNEL

$\mathrm{M}_{\infty}=0.4$

$0\left(\mathrm{~h}^{2}\right), 134 \times 70 \& 266 \times 138(\cdots)$

$\triangle$ Top wall, $O$ Bottom wall
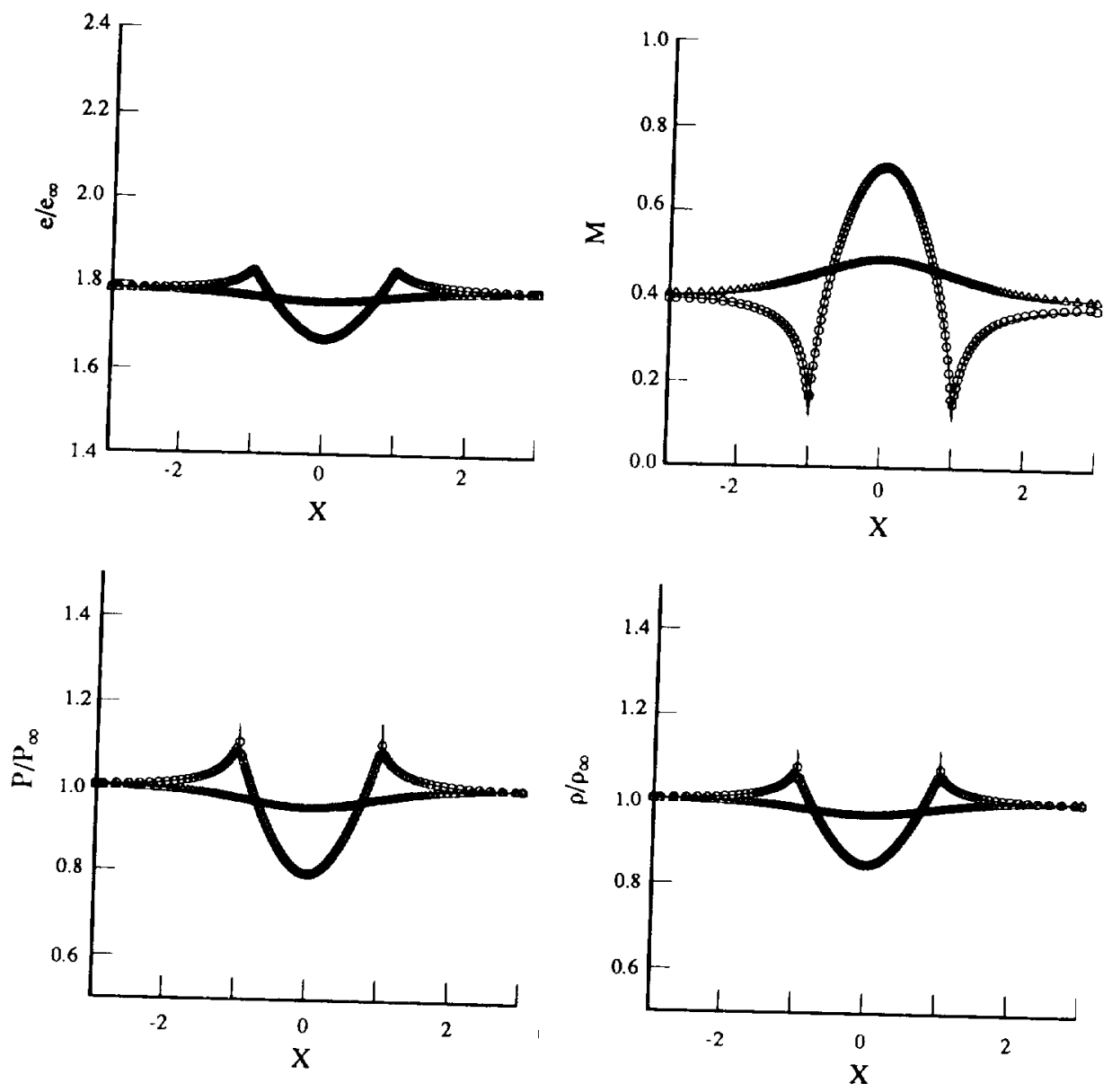
FIG. 5. Distributions of variables on top and bottom walls for $M_{x}=0.4,20 \%$ bump flow; lines are Eulerian solution (266 $\times 138$ grid) and symbols are
Lagrangian solution ( $134 \times 70$ grid) with $\Delta$ and $\odot$ denoting top and bottom walls, respectively.

with the instantaneous particle paths and $\partial \Omega_{V}^{\mathrm{EL}}$ represents the inflow and outflow faces.

Clearly, the present "extented Lagrangian method" combines the quality of the above two approaches: the second surface integral includes both convective and pressure terms, as in the case of Eulerian approach; the first surface integral, on the other hand, merely has the effect of pressure, as in the case of Lagrangian approach. That is, see Fig. 2,

on $\partial \Omega_{V}^{E L} \leftarrow$ both convected and pressure fluxes on $\partial \Omega_{P}^{\mathrm{EL}} \leftarrow$ only pressure flux

For steady flow (hence $\vec{V}_{h}=0$ ), there is no need for literally following particles because no variations of motion with time appear among the particles on the same streamline. Therefore the question whether we strictly follow particles of same identity or not is irrelevant in the formulation. Indeed, following the streamlines, rather than particles, is the essence of the present approach and this rescues us from facing the difficulty of other Lagrangian approaches. Substituting $\vec{V}_{b}=0$ in Eq. (11), we get

$$
\begin{aligned}
& \frac{d}{d t} \int_{\Omega^{\mathrm{EL}}} \mathbf{U} d v+\int_{\pi \Omega_{\mu}^{\mathrm{EL}}} \overrightarrow{\mathbf{P}} \cdot d \vec{S} \\
& +\int_{i J_{V}^{\mathrm{EL}}}\left[\mathbf{U}_{c} \vec{V}+\overrightarrow{\mathbf{P}}\right] \cdot d \vec{S}=0, \quad \text { in } \Omega^{\mathrm{EL}} .
\end{aligned}
$$

together with the constraint, 


$$
\vec{V} \cdot \vec{S}=0, \quad \text { on } \partial \Omega \Omega_{P}^{\mathrm{EL}} .
$$

The semi-discrete form, including the time-dependent term retained for iteration purpose, can be cast as:

$$
\frac{d}{d t} \int_{\Omega^{\mathrm{EL}}} \mathbf{U} d v+\sum_{i \in i s \Omega_{p}^{\mathrm{EL}}} \overrightarrow{\mathbf{P}}_{i} \cdot \vec{S}_{i}+\sum_{i \in d \mathrm{I} \Omega_{v}^{\mathrm{EL}}} \overrightarrow{\mathbf{F}}_{i} \cdot \vec{S}_{i}=0
$$

Examination of the above equations reveals some interesting insight. The inbalance of pressure in two neighboring cells with common interface boundary $\partial \Omega_{P}^{\mathrm{EL}}$ (e.g., $\vec{S}_{i, j}$ in) causes the change of flow direction (i.e., $\vec{V}$ in $\mathbf{U}_{i, j}$ ) of the fluids under consideration as well as change of their volumes $\left(v_{i, j}\right)$. In other words, the deformation and dilatation of the fluid can be described. Indeed, the Lagrangian grid includes multi-dimensional information and suggests how the fluid volume distorts in the flow. This point of view makes the description of fluid motion intuitively simple and clear. Moreover, it results in a major benefit in the numerical solution because this formulation avoids any arbitrary (numerical) mixing of fluids which in turn introduces numerical diffusion in the solution, notably across the contact discontinuity or shear layer. This diffusion error is common in the Eulerian formulation in which a nonstationary contact discontinuity is smeared without bound as time/space is marched. Furthermore, the advantage of the present approach is also clearly shown in its capability for crisply resolving multi-dimensional shocks.

We note that the steady form of Eq. (13) is what the streamline-coordinate method solves, see, e.g., $[17,18]$. Since the equation is valid only in the streamline coordinate, streamlines (thus grid lines) must be compatible with and be part of the solution. As a result, the number of unknowns is increased, leading to substantial increase in computation effort. Further, the effect of grids on the solution during the iterative process is strongly felt, robustness is of concern. Thus it is important to have a reasonably good initial position for the streamlines [18].

In the present extended Lagrangian method, we are only interested in requiring that the limit form of Eq. (5) under the constraint, Eq. (10) - namely Eqs. (11) or (13) - be satisfied at convergence to unsteady or steady solutions. During intermediate steps or any other times, it is not required that Eqs. (11) or (13) be solved. In other words, the equation to be solved for the present method is Eq. (5). The grid as well as conservation equations are not assumed to be in the streamline coordinate and the fluxes have the same form on every face of a cell. The grid instead evolves during the iteration according to Eq. (10) when it is invoked, resulting in a very simple and negligible effort to do. Since the grid is only a result of the solution and there is enough flexibility for adjustment hence goodness of initial grid or robustness is not an issue.
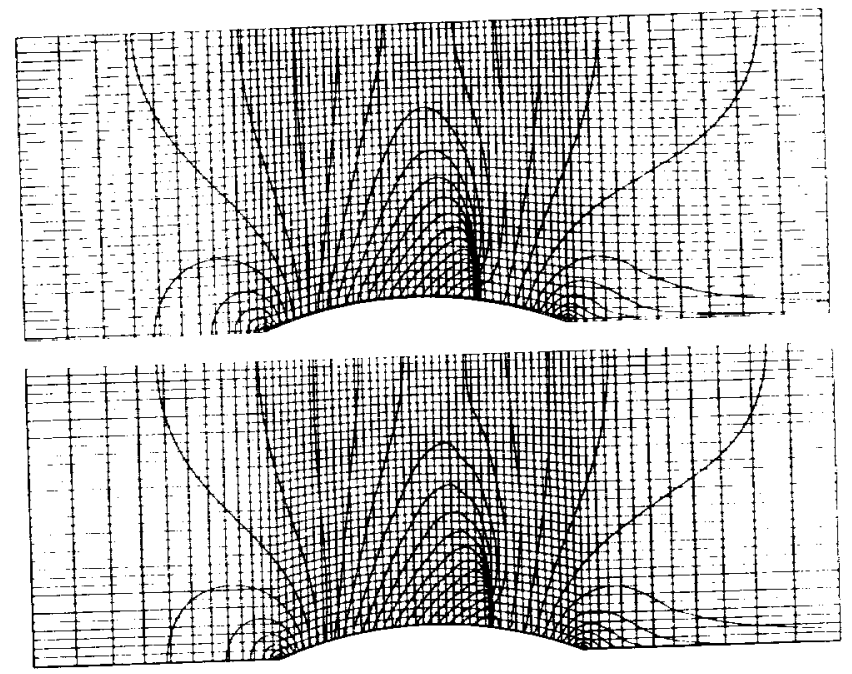

FIG. 6. Mach contours for $M_{x}=0.675,10 \%$ bump flow; (a) Lagrangian solution $(134 \times 70$ grid), (b) Eulerian solution $(266 \times 138$ grid). (Contour

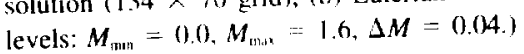

We now conclude: the grid constraint EL expressed in Eq. (10) is all it takes to produce the extended Lagrangian method from an existing Eulerian method. In other words, ALE method or any other Eulerian codes can adopt Eq. (10) to arrive at the present method, leaving the existing code intact.

In practice, the convective flux does not vanish exactly because of the interpolation approximation used in following the constraint EL. However, the error, namely the deviation from "zero" measured by the ratio of the convective and pressure fluxes on the transverse faces, $\max _{i, j}\left\|\mathbf{U}_{c} \vec{V} \cdot \Delta \vec{S}\right\| /\|\overrightarrow{\mathbf{P}} \cdot \Delta \vec{S}\|$, is negligible-on the order of $10^{-7}$ for the $M=0.4$ case, discussed later in Section 8.

We turn now to describe how Eq. (10) is fulfilled numerically.

\section{MOTION OF LAGRANGIAN GRIDS}

An important integral part of the present method is the grid motion that follows the constraint Eq. (10) or (12b) for steady flows. Two basic settings can be chosen for defining the motion of computational cells, namely the motion of cell centers or cell vertices. With the former approach the cell vertices will be defined by the position of neighboring centers, vice versa for the latter. Since the constraint, Eq. (12b), is imposed on the cell boundary, it is consistent to determine the vertex motion instead. This is easily done with the velocity field known from the solution. The constraint Eq. (12b) is equivalent to the kinematic condition on a streamline:

$$
\frac{d x}{u}=\frac{d y}{v}=\frac{d z}{w}
$$

Again we remind the reader that a more general constraint is 


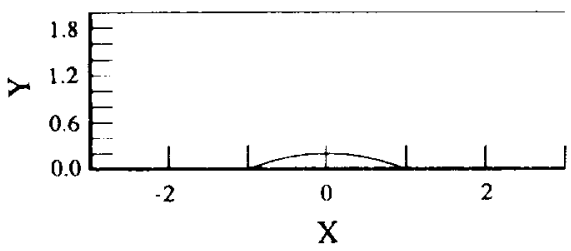

$10 \%$ BUMP ON A CHANNEL

$\mathrm{M}_{\infty}=0.675$

$0\left(h^{2}\right), 134 \times 70 \& 266 \times 138(-\cdots-\cdot)$

$\triangle$ Top wall, $\bigcirc$ Bottom wall
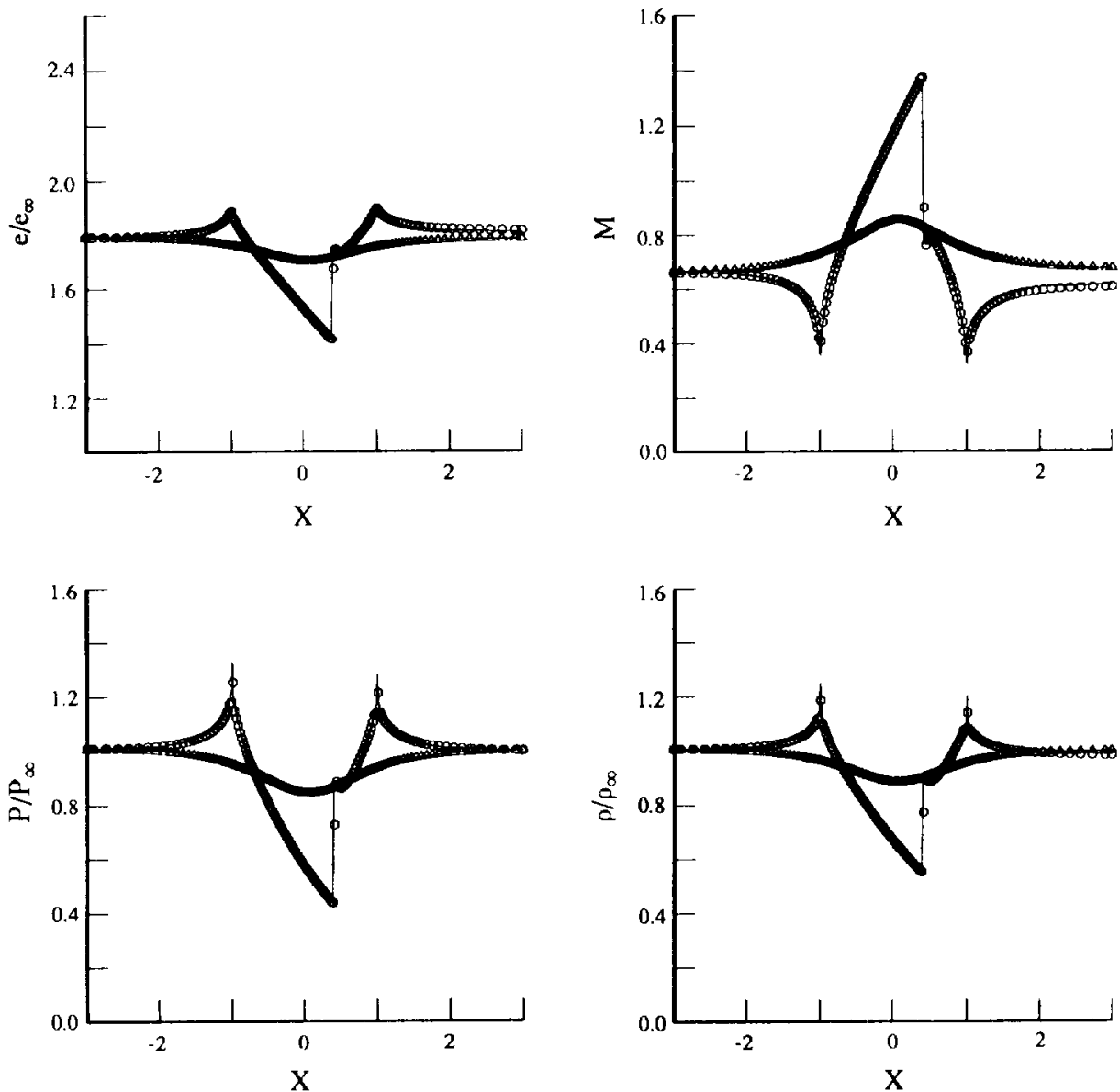

FIG. 7. Distributions of variables on top and bottom walls for $M_{x}=0.675,10 \%$ bump flow; lines are Eulerian solution (266 $\times 138$ grid) and symbols are Lagrangian solution ( $134 \times 70$ grid) with $\Delta$ and $\odot$ denoting top and bottom walls, respectively.

$$
\frac{d x}{u-u_{h}}=\frac{d y}{v-v_{b}}=\frac{d z}{w-w_{b}}
$$

where $\vec{V}_{b}$ can be chosen in a prescribed way that the cell remain regular, thereby eliminating grid tangling.

As flow variables are defined at cell centers, the velocity components at cell boundary must be defined by some interpolation procedure from surrounding cells. In the present report, we outline the general notion of grid movement and give a specific strategy showing how the grid is moved to meet the constraint for the test cases included in the paper. Let us consider the two-dimensional cell $(i, j)$, shown in Fig. 3 . Three- dimensional cells can be treated similarly. Assuming the cell boundary is described by a line segment $\left(\vec{r}_{i+1, j+1}-\vec{r}_{i, j+1}\right)$. Since the segment is a part of a streamline, Eq. (14) gives

$$
y_{i+1, j+1}=y_{i, j+1}+\bar{m}\left(x_{i+1, j+1}-x_{i, j+1}\right)
$$

where

$$
\bar{m}=\frac{\bar{v}}{\bar{u}}
$$

Here we list some possibilities for evaluating $(\bar{u}, \bar{v})$ : 

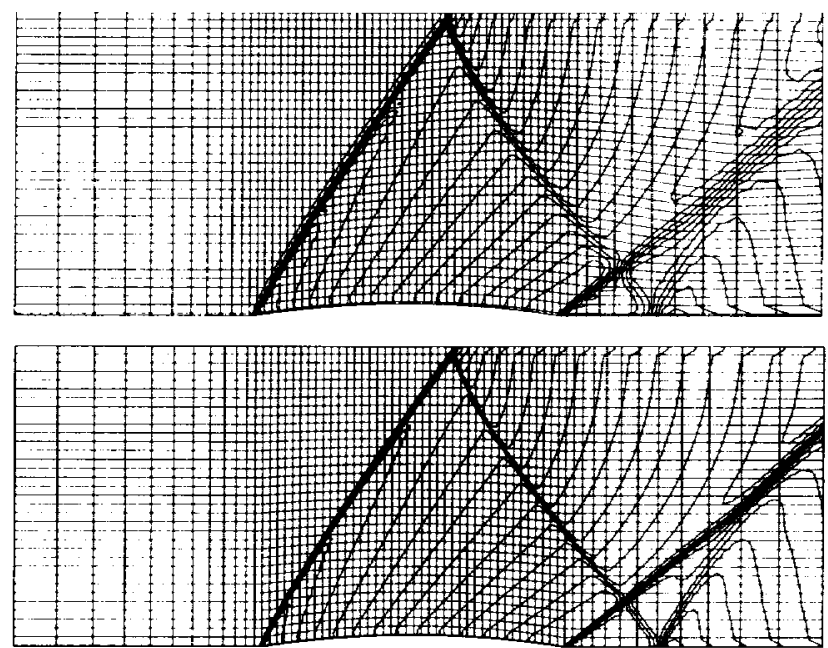

FIG. 8. Mach contours for $M_{\infty}=1.4$, $4 \%$ bump flow; (a) Lagrangian solution $(134 \times 70$ grid), (b) Eulerian solution $(266 \times 138$ grid). (Contour levels: $M_{\text {rux }}=0.4, M_{\text {max }}=2.2, \Delta M=0.045$.)

\section{(a) Mid-point average}

$$
\overline{\vec{V}}=\frac{1}{8}\left[2 \vec{V}_{i, j}+2 \vec{V}_{i, j+1}+\vec{V}_{i+1, j}+\vec{V}_{i+1 . j+1}+\vec{V}_{i 1, j}+\vec{V}_{i-i, j+1}\right],
$$

(b) Upstream average, assuming $u_{t, j}>0, \forall i, j$,

$$
\overline{\vec{V}}=\frac{1}{4}\left[\vec{V}_{i, j}+\vec{V}_{i, j+1}+\vec{V}_{i-1, j}+\vec{V}_{i, j+1}\right]
$$

There are two unknowns, $(x, y)_{i+1, j+1}$, in Eq. (16). Another condition is needed to complete the system. In this report, we prescribe the value of $\mathrm{x}$-coordinate for each $i$ th grid line, i.e., $x=$ constant lines. This condition provides simplicity, but also yields accuracy as will be shown later. Furthermore, specification of $\mathrm{x}$-coordinate allows one to put fine grids to resolve geometry details, e.g., near high curvature region.

When the constraint, Eq. (12b), is satisfied for all $j$ th grid line, the conservation laws are basically solved in a one-dimensional stream tube, because there is no flow across the $j$-grid lines. As a result, high accuracy is expected with this virtually onedimensional problem, which is demonstrated by the sharp representation of oblique shocks, as seen in Section 8 to be as accurate as their counterpart in one-dimension. The reason is that the formulation in itself already inherits multi-dimensional information via the deformation of grid lines (i.e., streamlines).

The cost of arriving at the above constraint is negligible even if it is done at each iteration, because calculation of Eqs. (16) and (17) is all it needs. The grid motion can be predicted in phase with the evolution of the flow variables, or else for a prescribed number of flow variables iterations. As for steady flow calculations, it is not necessary to adjust the grid so frequently while the flow is still evolving. A more thorough investigation about the optimal number of iterations per Lagrangian grid motion, its sensitivity to flow condition, and the effect of the velocity-averaging formulas will be useful, but is beyond the scope of the present paper. At this juncture, we note that for the results included in this paper, we used the mid-point average formula, Eq. (17a).

To complete the numerical solution procedure, we define the numerical fluxes by employing the newly developed upwind scheme AUSM, which is described in full detail in [22, 23]. In what follows, we shall see that this new splitting has a very interesting bearing with the present extended Lagrangian method.

\section{THE AUSM UPWIND METHOD $[22,23]$}

To illustrate the concept, it is sufficient to consider only the one-dimensional system. As a first step, by recognizing convection and pressure as two physically distinct (but coupled) processes, we split the flux in the form of Eq. (3). In other words, these two terms deserve separate treatments. Mathematically, we propose to separately deal with the genuinely nonlinear $((u-a, u+a)$ pair $)$ and linearly degenerate $(u)$ fields.

$$
\mathbf{F}=u\left(\begin{array}{c}
\rho \\
\rho u \\
\rho h_{\imath}
\end{array}\right)+\mathbf{P}=\mathbf{F} c+\mathbf{P}, \quad \mathbf{F}_{c}=u \mathbf{U}_{\iota}
$$

The overhead arrow "..," has been dropped for we are concerned only with one-dimensional flow. Both Mach number and velocity splittings can be used to represent the convective quantity $u$ in $\mathbf{F}_{c}$. In most cases, there is virtually no difference between calculated results of the two splittings. As found in [23], the velocity splitting is more robust in calculating unsteady shock tube problems by allowing a larger time step at start. Now, the numerical convective flux at the interface (denoted by subscript $1 / 2)$ straddling the left $(L)$ and right $(R)$ states, is effectively written as

$$
\mathbf{F}_{\mathrm{c} / 2}=\boldsymbol{u}_{1 / 2} \mathbf{U}_{\mathrm{cL} / \mathrm{R}},
$$

where $u_{1 / 2}$ is the interface convective velocity. Let $u_{1 / 2}$ be written as:

$$
u_{1 / 2}=u_{\mathrm{L}}^{+}+u_{\mathrm{R}}^{-} .
$$

Several formulas are appropriate to define $u^{ \pm}$, e.g.,

$$
u^{*}=\left\{\begin{array}{ll}
(u \pm|u|) / 2, & \text { if }|u| \geq a \\
\pm(u \pm a)^{2} / 4 a, & \text { otherwise }
\end{array} .\right.
$$




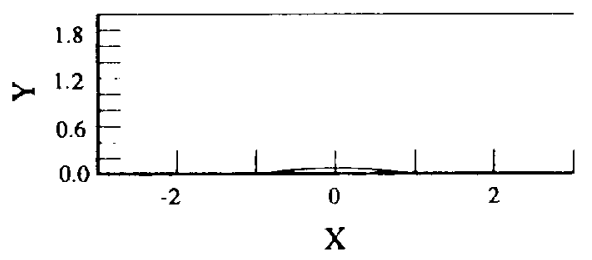

$4 \%$ BUMP ON A CHANNE1

$\mathrm{M}_{\infty}=1.4$

$0\left(h^{2}\right), 134 \times 70 \& 266 \times 138(\cdots)$

$\triangle$ Top wall, $O$ Bottom wall
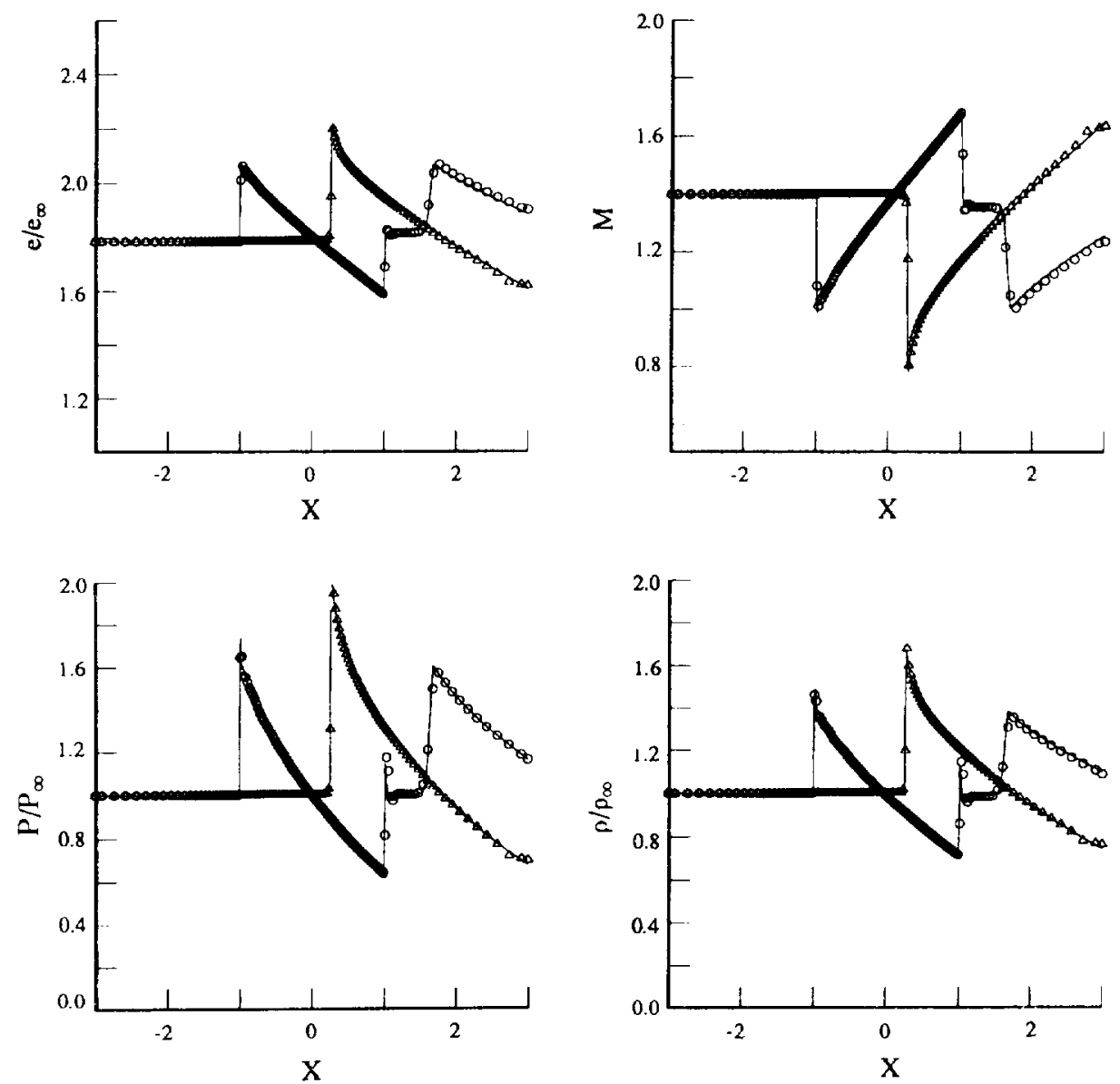

FIG. 9. Distributions of variables on top and bottom walls for $M_{x}=1.4,4 \%$ bump flow; lines are Eulerian solution $(266 \times 138$ grid $)$ and symbols are Lagrangian solution $(134 \times 70$ grid) with $\Delta$ and $\odot$ denoting top and bottom walls, respectively.

where $a$ is the speed of sound. The convectible variable vector $\mathbf{U}_{\text {c }}$ is then upwinded solely based on the sign of $u_{12}$, viz,

$$
\mathbf{U}_{c / / R}= \begin{cases}\left(\mathbf{U}_{c}\right)_{L}, & \text { if } u_{1 / 2} \geq 0, \\ \left(\mathbf{U}_{c}\right)_{R}, & \text { otherwise, }\end{cases}
$$

We turn now to the pressure term by writing:

$$
p_{1 / 2}=p_{\mathrm{l}}^{+}+p_{\mathrm{R}} .
$$

Similarly, a whole host of choices are possible for the pressure splitting. A differentiable pair of the ' + ' and '- ' components have been found to be effective.

$$
p^{ \pm}= \begin{cases}p(1 \pm \operatorname{sgn}(u)) / 2, & \text { if }|u| \geq a, \\ p(M \pm 1)^{2}(2 \mp M) / 4, & \text { otherwise. }\end{cases}
$$

This completes the definition of the numerical flux $\mathbf{F}$. Putting (19) and (23) together, we recast the interface flux in the following form

$$
\mathbf{F}_{1 / 2}=u_{1 / 2} \frac{1}{2}\left(\mathbf{U}_{\mathrm{c} L}+\mathbf{U}_{\mathrm{cR}}\right)-\frac{1}{2}\left|u_{1 / 2}\right| \Delta_{1 / 2} \mathbf{U}_{c}+\mathbf{P}_{1 / 2} .
$$



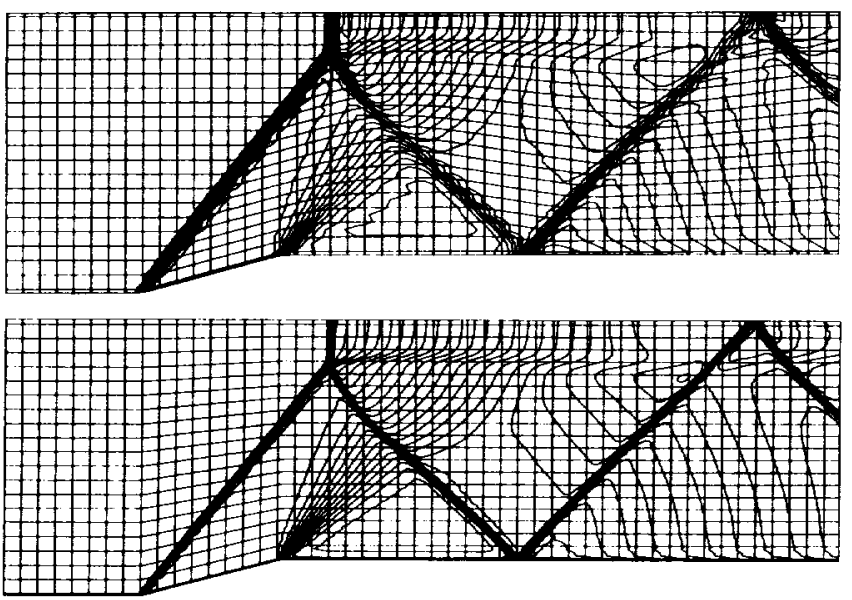

FIG. 10. Mach contours for $M_{\infty}=1.8,15 \%$ ramp flow; (a) Lagrangian solution $(100 \times 41$ grid), (b) Eulerian solution (198 $\times 80$ grid). (Contour levels: $M_{\min }=0.4, M_{\max }=2.2, \Delta M=0.045$.)

where $\left.\Delta_{1 / 2} \mid \cdot\right\}=\{\cdot\}_{R}-\{\bullet\}_{L}$. Here the first term on the RHS is clearly not a simple average of the ' $L$ ' and ' $R$ ' fluxes, but rather a weighted average via the convective velocity. The dissipation term has merely a scalar coefficient $\left|u_{1 / 2}\right|$ and requires only $O(n)$ operations for an $n$-dimensional vector F. Furthermore, since there is no differentiation (or jacobian matrix) involved in evaluating $\mathbf{F}_{1 / 2}$, the present method is easily extended to a general equation of state and non-equilibrium flows and the cost is only linearly increased with the additional conservation equations considered. Unlike the Roe or Osher schemes, the extension does not yield additional ambiguity such as the definition of averaged or intermediate states. Also, numerical tests strongly suggest an entropy-satisfying property by the present method.

To achieve higher-order spatial accuracy, a MUSCL-type procedure is followed to upwind-extrapolate variables (primitive variables in the calculations presented in this paper), with TVD limiters incorporated [24]. Then, a two-stage RungeKutta procedure is used to integrate the semi-discrete system Eq. (13), subject to the kinematic condition Eq. (12b).

The subsonic inflow conditions are imposed by specifying total enthalpy, total pressure, and flow angle, while the outflow conditions are obtained with specified static pressure and extrapolated total enthalpy, total pressure, and flow angle. The usual tangency procedure is used at the cell boundary that coincides with a physical wall - no ghost cells are used. The wall pressure is gotten using linear extrapolation from interior data, and so is the total enthalpy.

\section{TEST PROBLEMS AND DISCUSSION}

We will show examples for flows at all speed regimes, featuring solution accuracy and grid aspects. The plots are organized uniformly for all cases presented. We show Mach contours overlaid on the grid used. Fine grids, by doubling grid number in both directions, are used only in the Eulerian calculation for comparison purpose. There are 80 and 160 cells on the bump respectively for the coarse and fine grids. The grids shown are only one half of the whole grid in the Lagrangian case, and one quarter in the fine-grid case, thus corresponding to roughly the same location in both plots. The symbols denote the entended Lagrangian solutions and the lines are the Eulerian solutions. The Mach contours are chosen for presentation so that any numerical anomaly or inaccuracy can be more easily depicted than the other variables such as pressure.

The first example is the purely subsonic flow in which an $M_{\infty}=0.4$ flow enters a channal with $20 \%$ circular bump, see Fig. 5 for detailed geometry. The Mach contours, given in Fig. 4 , show nearly perfect symmetry about the midchord, except in the wake region. The wake region suggests an entropy production (numerical diffusion), likely due to numerical wall boundary condition, which to my knowledge is still a gray area in CFD. The Lagrangian solution appears to result in a narrower numerical wake, as indicated in the shaded area; the Lagrangian result is roughly half the width of the Eulerian result. Moreover, close examination of these plots reveals that the numerical wake of the Lagrangian solution does not spread while on the contrary the wake of the Eulerian solution grows with distance. It is worth noting that the present grid automatically evolves from initial Eulerian grid into the grid system seen in Fig. 4, according to Eqs. (16) and (17a). The grid spacing between streamlines increases near the stagnation points and converges as flow accelerates. The detailed distribution of variables on the top and bottom walls are plotted in Fig. 5. The fine-grid Eulerian solutions are included for comparison. The agreement is remarkable and again symmetry is quite evident. However, the fine-grid Eulerian solution over-predicts the pressure at the leading and trailing stagnation points, whose theoretical value is 1.116 , while the Lagrangian solution closely matchesmainly due to the expansion and contraction of grid lines near these two points with the largest change in slope. Hereafter, for briefness and contrast to the Eulerian solution, we shall take the liberty of loosely using the term "Lagrangian solution" to mean the solution obtained by the extended Lagrangian method described in this paper-not in the strict Lagrangian sense. It is also noted that the fine-grid solution took considerably more iterations than the Lagrangian solution to converge.

The second example involves the popular test of transonic flow in a channel with $10 \%$ bump and $M_{\infty}=0.675$. Results are given in Figs. 6 and 7. Again, we show the Mach contours and distributions on both walls. The agreement of the coarsegrid Lagrangian solutions with the fine-grid Eulerian solutions is excellent. The shock resolution from the Lagrangian method is outstanding, so is the prediction of the so-called "Zierep" singularity at the foot of the shock on the curved surface. The Lagrangian solution again yields a grid that senses the global flow characteristic. Notice that no clustering of grid is necessary to capture the shock in the correct location. 


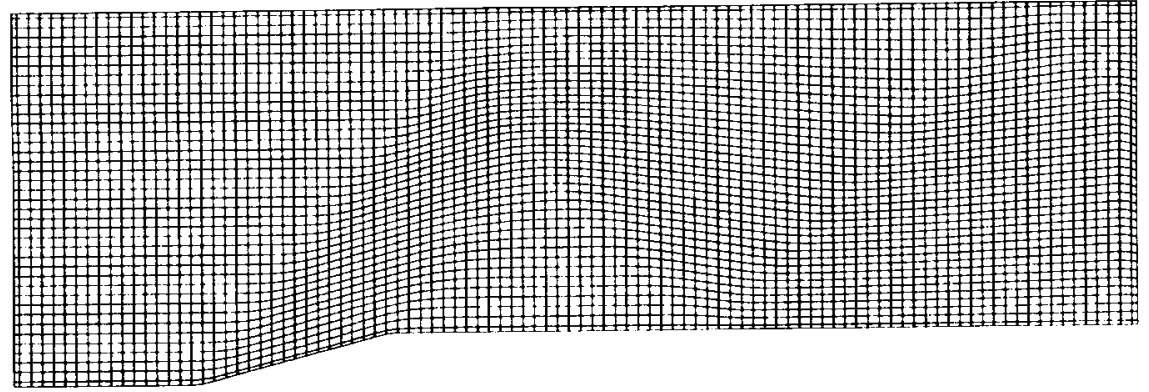

FIG. 11. Lagrangian grid, showing streamlines and the trains of shock and expansion waves.

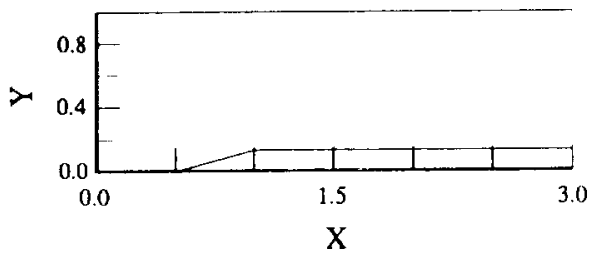

15-Deg RAMP IN A CHANNEL,

$M_{\infty}=1.8$

$0\left(\mathrm{~h}^{2}\right), 100 \times 41 \& 198 \times 80(\cdots)$

$\triangle$ Top wall, $O$ Bottom wall
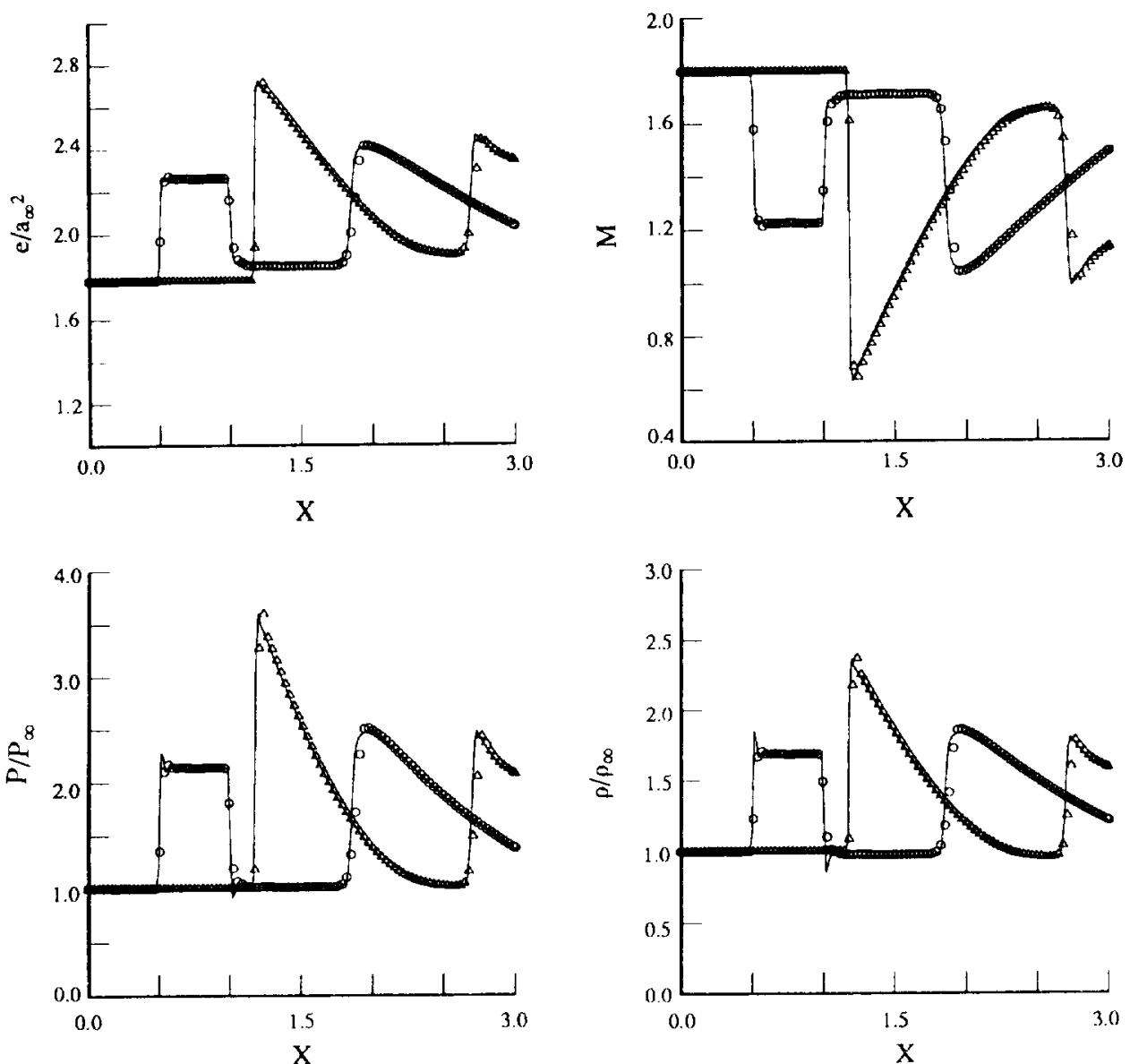

FIG. 12. Distributions of variables on top and bottom walls for $M_{x}=1.8,15^{\circ}$ ramp flow: lines are Eulerian solution (198 $\times 80$ grid) and symbols are Lagrangian solution $(100 \times 41$ grid $)$ with $\Delta$ and $\odot$ denoting top and bottom walls, respectively. 
The next case is also a standard one, involving an $M_{\infty}=1.4$ flow and $4 \%$ bump. This case consists of small subsonic pocket resulting from short Mach stems on both walls, shown in Fig. 8. Shock-shock-expansion waves interactions take place behind the trailing edge. The shock locations are in excellent agreement with the fine-grid solutions. Close examination of the Lagrangian grid shows that the grid lines (also streamlines) remain straight until the shock is encountered, as it should. The Eulerian grid however already began bending at the leading edge for all $j$ th lines, simply because of geometry constraint. The present method yields grids that are conforming with the flow features by bending, expanding/contracting. The net result is that excellent shock resolution is obtained, even though the shock is oblique to the grid line. Figure 9 displays a one-cell capturing of the oblique shock. This is not entirely surprising since the present formulation has already taken account of the multi-dimensional nature of the flow via the streamline deformation caused by the fluxes (pressure forces) of surrounding fluids.

The fourth test is an $M_{\infty}=1.8$ flow over a $15^{\circ}$ ramp. This case consists of a Mach stem about $10 \%$ of channel height, a slip line emanating from the triple point, and reflected shocks. In Fig. 10, the mach contours depict an overall picture of the flow, demonstrating a sharp resolution of the ramp shock, Mach stem, and the subsequent shocks. The slip line, whose strength is being weakened by the expansion wave generated at the ramp shoulder and transmitted through the first reflected shock, is resolved to the same level of accuracy as given by the finegrid solution, i.e., with the same level of spatial spreading. Figure 11 vividly displays how the grid lines bend as the shock is encountered and change direction according to the flow. The grid itself already suggests the flow structures, train of shock reflections, expansions, as well as the Mach stem across which there is no change of flow angle. It is worth noting the clear slipline emanating from the triple point. In contrast to the shockaligned grid, the present grid is aligned with the streamlines, which will never cross each other, but the shocks can. Thus the present method is indifferent to whether the high-gradient regions intersect. The profiles (Fig. 12) on the walls show good agreement of both solutions. Excellent shock resolution capability is observed on both walls even the second reflected shocks remain well resolved.

\section{CONCLUDING REMARKS}

We have presented a unique formulation for dealing with subsonic as well as supersonic flows. The method, referred to as extended Lagrangian method, combines the accuracy belonging to a Lagrangian description and the robustness and simplicity of an Eulerian description. Through systematic comparison with fine-grid solutions and a theoretical check for flows at various regimes, we have demonstrated its capability for crisply capturing high-gradient regions that are not aligned with the grid. In contrast to adaptive approaches reported to date, the present approach already automatically inherits the ability to adapt to flow features, but based on an entirely different adaptive philosophy in which there is no need for clustering grid lines. Since the grid lines are not aligned with high-gradient areas, they are maintained regular and uniform. Moreover, one set of grid lines that coincides with streamlines depicts vividly a form of "numerical flow visualization." Without resorting to arbitrary detecting criteria, the present approach not only predicts well the nonlinear waves, such as shock and rarefaction waves, but also is especially amenable to treating a linearly degenerate field, such as a contact discontinuity. Furthermore, since the grid spacing is maintained relatively unform, a large time step is permitted throughout the calculation, thus increasing efficiency. Also the common adaptive strategy will have great difficulty in the case of intersecting shocks, because the grid lines will cross each other, if not checked. We also suggest that the present extended Lagrangian method is a viable alternative approach to the current multi-dimensional scheme and gridenriching adaptive procedure for complex flows having highgradient regions. Further development and 3D applications are currently underway and will be reported in the future.

\section{ACKNOWLEDGMENTS}

I thank the referees for suggesting valuable and constructive comments which have been incorporated in the revision and led to improvements of the paper.

\section{REFERENCES}

1. S. K. Gudonov, Mat. Sb. 47, 271 (1959).

2. C. Y. Loh and W. H. Hui J. Comput. Phys. 89, 207 (1990).

3. C. Y. Loh and M.-S. Liou, J. Comput. Phys. 104, 150 (1993).

4. C. Y. Loh and M.-S. Liou, J. Comput. Phys. 113, 224 (1994).

5. C. W. Hirt, A. A. Amsden, and J. L. Cook, J. Comput Phys. 14, 227 (1974).

6. W. Pracht, J. Comput. Phys. 17, 132 (1975).

7. J. K. Dukowicz, and B. J. A. Meltz, J. Comput. Phys. 99, 115 (1992).

8. F. H. Harlow and A. A. Amsden, J. Comput. Phys. 8, 197 (1971).

9. A. Harten, J. Comput. Phys. 83, 148 (1989).

10. F. L. Addessio, et al., "CAVEAT: A Computer Code for Fluid Dynamics Problems with Large Distortion and Internal Slip," Los Alamos National Laboratory, LA-10613-MS, 1986.

I1. A. A. Amsden, P. J. Rourke, and T. D. Butler, "KIVA-II: A Computer Program for Chemically Reactive Flows with Sprays," Los Alamos National Laboratory, LA-11560-MS, 1989.

12. W. H. Hui and H. Van Roessel, "Unsteady Three-Dimensional Flow Theory via Material Functions," in AGARD Symposium on Unsteady Aerodynamics - Fundamentals and Application to Aircraft Dynamics, Götting. West Germany, 1985, S1, CP-386.

13. W. H. Hui and Y. C. Zhao, "A Generalized Lagrangian Method for Solving the Euler Equations," in Proceedings of the 4th International Conference on Hyperbolic Problems, (edited by A. Donato and F. Oliveri) (1993).

14. R. A. Novak, J. Eng. Power, October, 478 (1967).

15. C.-H. Wu and B. Wang, J. Eng. Gas Turbine Power 106, 449 (1984).

16. J. S. Keith, D. R. Ferguson, C. L. Merkle, P. H. Heck, and D. J. Lahti, "Analytical Method for Predicting the Pressure Distribution about a Nacelle at Transonic Speeds," NASA CR-2217, July 1973 (unpublished). 


\section{EXTENDED LAGRANGIAN METHOD}

17. M. B. Giles and M. Drela, AIAA J. 25, 1199 (1987).

18. M. B. Giles, "Newton Solution of Steady Flow," GTL Report 186, Gas Turbine Laboratory, Mimensional Transonic lished). $\quad 1985$ (unpub-

19. A. H. Shapiro, Compressible Fluid Flow, Vol. I (Wiley,

20. H. W. Liepmann and A. Riley, New York, 1953). York, 1957). 1972), p. 15

23. M.-S. Liou, "On C. J. Steffen, J. Compus. Phys. 107, 23 (1993),

Vol. 414, (Springer-Verlag

24. M.-S. Liou and A. T. Hsug, Berlin/New York, 1993), p. 115.

tion Scheme for Three Dimensionale Accurate Finite Volume High ResoluPaper 89-1994-CP, 1989. 
- 